\title{
Reliability of lung crackle characteristics in cystic fibrosis and bronchiectasis patients in a clinical setting
}

\author{
Alda Marques ${ }^{1}$, Anne Bruton $^{2}$ and Anna Barney ${ }^{3}$ \\ ${ }^{1}$ Escola Superior de Saúde, Universidade de Aveiro, Campus Santiago Edifício II, 3810-19,3 Aveiro, \\ ${ }^{I}$ Portugal, amarques@ua.pt \\ ${ }^{2}$ School of Health Sciences, University of Southampton,Building 45, Highfield Campus, Southampton, \\ SO17 1BJ, United Kingdom, ab7@ soton.ac.uk \\ ${ }^{3}$ Institute of Sound and Vibration Research, University of Southampton, Highfield Campus, Southampton, \\ SO171BJ, United Kingdom, ab3@soton.ac.uk
}

\begin{abstract}
Lung sounds provide useful information for assessing and monitoring respiratory patients, but standard auscultation is subjective. Computer Aided Lung Sound Analysis (CALSA) enables the quantification and characterisation of added lung sounds (e.g. crackles). At present, little is known about the reliability of these sound characteristics. Therefore, the aim of this study was to explore the reliability of crackle Initial Deflection Width (IDW) and Two Cycles Deflection (2CD) in a clinical population. Fifty-four subjects (37 bronchiectasis; 17 cystic fibrosis) were recruited from out-patient clinics. Three repeated lung sound recordings were taken at 7 anatomical sites with a digital stethoscope connected to a laptop computer. The intra-subject reliability of crackle IDW and $2 \mathrm{CD}$ was found to be 'good' to 'excellent', estimated by the Analysis of Variance, Intraclass Correlation Coefficient (IDW 0.76;0.85; 2CD 0.83;0.94), Bland and Altman 95\% limits of agreement (IDW $-0.50 ; 0.47 \mathrm{~ms} ; 2$ CD $-2.12 ; 1.87 \mathrm{~ms}$ ) and Smallest Real Difference (IDW 0.30;0.66ms; 2CD 1.57;2.42ms). Crackle 2CD was found to be more reliable than IDW. It is concluded that crackle IDW and 2CD characterized by CALSA have good test-retest reliability. This technique requires further evaluation since CALSA has potential to diagnose or monitor respiratory conditions, and provide an objective physiological measure for respiratory interventions.
\end{abstract}

Keywords: crackles, lung sounds, reliability

\section{Introduction}

Sounds generated in the lungs should relate directly to airway geometry, the movement of air and the presence of secretions. Computer aided lung sound analysis (CALSA) is designed to access some of the information that is not possible using standard auscultation techniques, by removing subjectivity and allowing lung sounds to be quantified and characterized. CALSA has previously been used as a diagnostic tool (Piirila et al., 1991, Murphy et al., 2004), and has recently been proposed as a potential outcome measure for respiratory interventions such as airway clearance therapy (Marques et al., 2006). However, there are few published data relating to the reliability of added lung sounds' characteristics. As reliability is a fundamental requirement of any physiological measure that affects decision making (Finch et al., 2002), this study aimed to assess the test-retest reliability of two of the characteristics (i.e. Initial Deflection Width (IDW) and Two-cycle deflection (2CD)) of one type of added lung sounds (i.e. crackles) in a clinical population.

Guidelines for recording and analysing lung sounds have been published to standardize terminology and techniques (Sovijarvi et al., 2000b). Crackles are intermittent, non-musical and brief sounds thought to be caused by the sudden opening of abnormally closed airways (Forgacs, 1978). The first recordings of crackles were presented in the 1970s, when Forgacs theorized that pulmonary crackles were generated during inspiration as a result of sudden opening of the airways (Forgacs, 1978). Despite decades of subsequent research this theory has

\footnotetext{
${ }^{1}$ Alda Marques: author to whom correspondence should be addressed.
} 
never been refuted. Currently, a crackle sound is believed to originate from the acoustic energy generated by pressure equalisation, resulting from a change in elastic stress after a sudden opening or closing of airways (Vyshedskiy et al., 2008, Nath and Capel, 1974), or when there is inflammation or oedema in the lungs (Kiyokawa et al., 2001). Crackles' short duration and, often, low intensity makes their discrimination and characterisation by standard auscultation difficult; and their detection becomes even more difficult when other breath sounds have greater intensity (Kiyokawa et al., 2001).

Crackles can be characterized by either the IDW, i.e. the duration of the first deflection of the crackle; the $2 \mathrm{CD}$, i.e. the duration of the first two cycles of the crackle or the Largest Deflection Width (LDW), i.e., width of the largest deflection of the crackle (see Figure 1). However, most sources only quote the mean values of IDW and 2CD durations for fine and coarse crackles. The American Thoracic Society (ATS, 1977) defines fine crackles as having an IDW of $0.7 \mathrm{~ms}$ and $2 \mathrm{CD}$ of $5 \mathrm{~ms}$; and coarse crackles as having an IDW of $1.5 \mathrm{~ms}$ and $2 \mathrm{CD}$ of $10 \mathrm{~ms}$. Another group have defined fine crackles as having a $2 \mathrm{CD}<10 \mathrm{~ms}$ and coarse crackles as having a $2 \mathrm{CD}>10 \mathrm{~ms}$ (Sovijarvi et al., 2000b). An increase or a decrease of the duration (ms), in both variables, means that crackles became longer or shorter, and therefore the frequency value decreases or increases, respectively. The association between crackle frequency and their origin in the airways has been explored by other authors (Fredberg and Holford, 1983). Smaller airways have been shown to produce late inspiratory crackles, shorter than $10 \mathrm{~ms}$ (perceived as high frequency), whereas larger airways tend to produce earlier crackles, with a duration longer than 10ms (perceived as low frequency) (Fredberg and Holford, 1983, Sovijarvi et al., 2000a). The presence of sputum in the peripheral airways, or the opening or closing of alveoli, will produce high frequency crackles; whereas sputum in the proximal airways will produce low frequency crackles. Therefore, through a greater understanding of the nature of recorded crackles, it should be possible to identify their source.

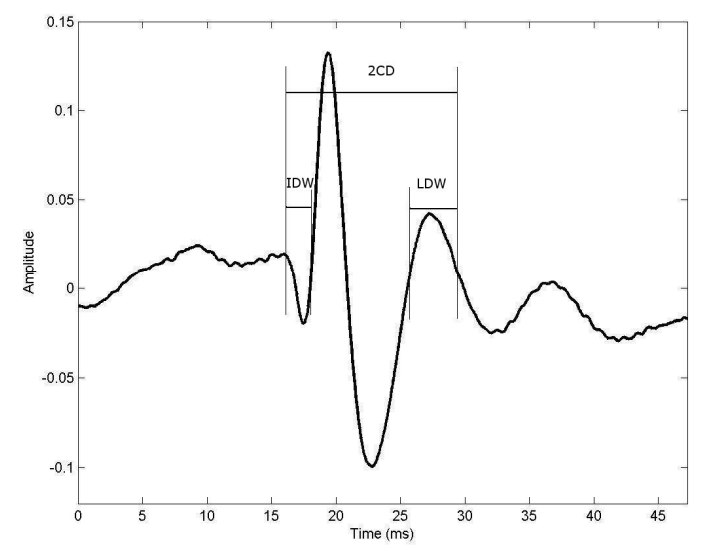

Figure 1. Crackle parameters. Values are in milliseconds (ms). IDW: Initial Deflection Width; 2CD: Two Cycles Deflection and LDW: Largest deflection Width.

Detailed crackle analysis could provide an objective, non-invasive, bedside physiological measure for monitoring respiratory conditions and assessing the effectiveness of therapies. However, in order for CALSA to be used as a valid and clinically useful physiological measure, its reliability within patient populations in clinical settings must be demonstrated. The aim of this study was to investigate the test-retest reliability over short time-periods of two crackle parameters (IDW and 2CD) using CALSA, in adult patients with bronchiectasis and cystic fibrosis.

\section{Methods}

\subsection{Study design}


A single group repeated measures design was employed in which triplicate recordings were made from the same patients when attending an outpatient clinic. The study received full approval from Southampton \& South West Hampshire Research Ethics Committees (A).

\subsection{Subjects and instrumentation}

The aim was to recruit around 50 subjects as Hopkins (Hopkins, 2000) has suggested that a sample of 30-50 are required to calculate a smallest real difference of practical use, and Bland and Altman (Bland and Altman, 1986) recommend a minimum sample of 50 for calculating 95\% limits of agreement. Potential subjects were identified via cystic fibrosis and bronchiectasis outpatient clinics held at a large general hospital on the south coast of the UK. Subjects were included in the study if they: 1) were able to give and sign informed consent; 2) had a documented diagnosis of cystic fibrosis or bronchiectasis; 3 ) were 18 years of age or older and 4) were clinically stable for one month prior to the study (no hospital admissions, exacerbations/infections or changes in medication). Subjects were excluded from the study if they had co-existing lung pathologies.

Anthropometric data (height and weight) were measured using calibrated digital scales. The lung sound recordings were performed with a digital stethoscope (Master Elite Sensor-based Stethoscope, WelchAllyn Meditron 5079-402) and lung function (Forced Expiratory Volume in the first second $\left(\mathrm{FEV}_{1}\right)$ and Forced Vital Capacity $(\mathrm{FVC})$ ) was measured using a spirometer (Micro Medical Microlab 3500). The input from the stethoscope microphone was connected via an integral amplifier to the sound card of a laptop with customized software, suitable for data acquisition and analysis, written in Matlab (version 7.1). A sampling frequency of $44.1 \mathrm{kHz}$ and a quantization of 16 bits were adopted for all recordings.

\subsection{Procedure}

All subjects provided informed consent prior to data collection. Demographic and basic anthropometric data were recorded first. The lung sound recordings followed the guidelines defined by the Computerized Respiratory Sound Analysis (CORSA) project for short term acquisition (Sovijarvi et al., 2000b). Subjects' skin was marked with a pen in seven different places to ensure consistency of stethoscope placement: one centrally over the trachea (on the sternal notch); two on the back of the chest (right and left bases: at $5 \mathrm{~cm}$ from the paravertebral line and $7 \mathrm{~cm}$ below the scapular angle); two on the front of the chest (right and left: in the second intercostal space, mid-clavicular line); two on the side of the chest (right and left: in the fourth to fifth intercostal space, mid axillary line). Lung sound recordings were performed with the digital stethoscope held manually over each location and subjects were asked to breathe through the mouth during recordings. Care was taken to ensure the stethoscope was kept as still as possible during all recordings to minimise added noise. While a more secure fixing for the stethoscope might have been achievable, our priority was to develop a measurement protocol sufficiently robust to be used within routine clinical practice and thus manual placement of the stethoscope head was preferable. Preliminary analysis suggested that the signature of a crackle, our focus in this study, was quite distinct from the more broadband additive noise that may occur due to, for example, friction between the stethoscope head and the body surface.

Three sets of recordings were made for 25 seconds at each marked location. Spirometry was then performed in accordance with published guidelines from the American Thoracic Society and the European Respiratory Society (Miller et al., 2005).

\subsection{Statistical Analyses}

Gender, date of birth, height, weight, $\mathrm{FEV}_{1}$ and FVC data were entered into SPSS version 14. Body mass index (BMI) in $\mathrm{kg} / \mathrm{m}^{2}$ was calculated using the formula $\mathrm{BMI}=$ weight $/(\text { height })^{2}$. Mean, standard deviation, minimum and maximum were used as descriptive statistics to characterize each parameter.

All data files from the seven anatomical sites (three recordings in each site), at each session, were processed using algorithms written in Matlab based on the algorithm developed by Vannuccini et al (Vannuccini et al., 1998). Various authors have developed automatic crackle detection (Kaisla et al., 1991, Murphy et al., 1989, Vannuccini et al., 1998). Although CORSA 
guidelines do not endorse a specific method, they do recommend band-pass filtering of between $60 \mathrm{~Hz}$ and $2 \mathrm{kHz}$ prior to crackle detection (Vannuccini et al., 2000). The algorithm developed by Vannuccini et al. follows Murphy et al.'s (Murphy et al., 1989) definition of crackles, has been validated using a sample of 200 inspiratory crackles recorded from 15 cryptogenic fibrosis alveolitis patients, and has been reported to show a sensitivity of $84 \%$ and specificity of $89 \%$ on their test data. Furthermore, the method provides a systematic way to identify the start of a crackle (Vannuccini et al., 1998) which was necessary to detect the crackle IDW and 2CD. In this research all recordings were performed by the same researcher. The mean and standard deviation of the IDW and 2CD duration of each recording, in each recording position, for each subject, was calculated and imported to SPSS version 14 for statistical analysis. To calculate inter-subject reliability the data for each of the three recordings for each subject and place was pooled and the average and standard deviation of IDW and 2CD were calculated. One-way ANOVA at 95\% significance level between the mean IDWs and 2CDs respectively for each subject at each place was carried out. For the intra-subject reliability analysis the mean of each of the three recordings for each subject and place was used. The intra-subject reliability was examined using the Intraclass Correlation Coefficient (ICC), which is a measure of relative reliability, Bland and Altman 95\% limits of agreement, and the Smallest Real Difference (SRD), which are measures of absolute reliability. These measures have been chosen because it has been suggested that to assess intra-rater (or test-retest) or inter-rater reliability in reliability studies, measures of relative reliability and absolute reliability should both be reported (Rankin and Stokes, 1998).

\subsubsection{Relative intra-subject reliability}

The ICC, was calculated using equation 1 (Fleiss, 1986) which uses the one-way ANOVA table, $\operatorname{ICC}(1, k)=(B M S-W M S) / B M S$

where ICC is the Intraclass correlation coefficient, $\mathrm{k}$ is the number of observers or measures (in this study $\mathrm{k}=3$ ), BMS is the between subjects mean squares and WMS is the within subjects mean squares.

The ICC results were analysed according to Fleiss (1986) criteria i.e. values above 0.75 represent 'excellent' reliability; between 0.4 and 0.75 represent 'moderate' to 'good' reliability and below 0.4 represent 'poor' reliability.

\subsubsection{Absolute intra-subject reliability}

Bland and Altman 95\% limits of agreement were used to assess agreement of the lung sounds recordings. The Bland and Altman techniques were developed for data with only two sets of measurements. Therefore, to reduce systematically the three data sets to two in each case, random numbers were generated in Excel and used to delete one of the three recordings in each place at random. The mean of the IDW and $2 \mathrm{CD}$ duration of the two remaining recordings, in each recording position, for each subject, were then used. For each of the IDW and 2CD two new variables were generated for each recording position across all subjects; the difference in the mean values between recordings and the grand average across recordings. Mean differences and their confidence intervals and 95\% limits of agreement were calculated and Bland-Altman plots were created for each variable in each recording position. These provide visual information as to systematic bias and random error by examining the direction and magnitude of the scatter around the mean difference (Bland and Altman, 1986). The standard error of measurement (SEM) was obtained by calculating the square root of the within subject mean square (WMS) values obtained in the one-way ANOVA (95\% significance level) table performed for each anatomical location for each session. These values were then used to calculate the SRD, which represents the smallest change that can be interpreted as a real difference as: $S R D=1.96 \sqrt{2}(\mathrm{SEM})$.

\section{Results}

As presented in Table 1, 54 subjects were recruited. They presented a range of demographic and anthropometric characteristics, pathology and acuity. Seventeen adults with cystic fibrosis (8 
female), aged 18 to 67 years and 37 adults with bronchiectasis ( 24 female), aged 25 to 83 years, were recruited.

Table 1. Characteristics of the cystic fibrosis $(\mathrm{CF})$ and bronchiectasis $(\mathrm{Br})$ subjects. Values are mean (SD). BMI: body mass index; $\mathrm{FEV}_{1} \mathrm{pp}$ : forced expiratory volume in 1 second percentage predicted; FVCpp: forced vital capacity percentage predicted.

\begin{tabular}{ccccccc}
\hline $\mathrm{n}$ & $\begin{array}{c}\text { Age (years) } \\
\text { Mean (SD) }\end{array}$ & $\begin{array}{c}\text { Weight } \\
(\mathrm{Kg})\end{array}$ & $\begin{array}{c}\text { Height }(\mathrm{m}) \\
\text { Mean }(\mathrm{SD})\end{array}$ & $\begin{array}{c}\text { BMI } \\
\left(\mathrm{Kg} / \mathrm{m}^{2}\right) \\
\text { Mean (SD) }\end{array}$ & $\begin{array}{c}\text { FEV } 1 \text { pp } \\
(\%) \\
\text { Mean (SD) }\end{array}$ & $\begin{array}{c}\text { FVC pp } \\
(\%) \\
\text { Mean (SD) }\end{array}$ \\
\hline CF- 17 & $29(13.3)$ & $58.5(15.7)$ & $1.67(0.2)$ & $20.6(3.3)$ & $46.1(18.2)$ & $58.1(13.8)$ \\
$\mathrm{Br}-37$ & $61(11.2)$ & $76.7(20)$ & $1.66(0.1)$ & $27.7(5.5)$ & $65.8(26)$ & $81.5(17.5)$ \\
\hline
\end{tabular}

Cystic fibrosis and bronchiectasis subjects presented with significantly different values for $\operatorname{BMI}(t(16)=-5.67, \mathrm{p}<0.001)$ and lung function $\left(\mathrm{FEV}_{1} \mathrm{pp}: t(16)=-4.07, \mathrm{p}<0.001 ; \mathrm{FVCpp}: t(16)\right.$ $=-4.91, \mathrm{p}<0.001)$. Reliability analysis of crackle characteristics was initially conducted separately using data from each pathological group. However, as the reliability results were found to be very similar for the two separate sub-groups and for the whole group, it was considered appropriate to present the results of all the subjects as a single group here.

The inter-subject reliability analysis (using ANOVA) showed significant variability $(\mathrm{p}<0.001)$ between subjects in all recording positions.

\subsection{Relative intra-subject reliability}

The ICC values are presented with their respective confidence intervals in Table 2. Calcuations are based on all 54 subjects. The ICC results were found to give 'excellent' reliability. The IDW generally presented a lower ICC value than the $2 \mathrm{CD}$.

Table 2. Relative reliability of the crackles' IDW and 2CD $(n=54)$. Values are from Intraclass Correlation Coefficient (ICC) with the 95\% Confidence Intervals (CI). T: trachea; AR: anterior right; AL: anterior left; LR: lateral right; LL: lateral left; PR: posterior right; PL: posterior left.

\begin{tabular}{ccc}
\hline & ICC $(95 \%$ CI $)$ IDW & ICC $(95 \%$ CI $) 2 C D$ \\
\hline T & $0.77(0.61 ; 0.87)$ & $0.94(0.90 ; 0.97)$ \\
AR & $0.76(0.59 ; 0.86)$ & $0.89(0.80 ; 0.93)$ \\
AL & $0.81(0.68 ; 0.89)$ & $0.83(0.70 ; 0.90)$ \\
LR & $0.83(0.70 ; 0.90)$ & $0.91(0.85 ; 0.95)$ \\
LL & $0.84(0.73 ; 0.91)$ & $0.87(0.78 ; 0.93)$ \\
PR & $0.82(0.70 ; 0.90)$ & $0.91(0.84 ; 0.95)$ \\
PL & $0.85(0.73 ; 0.91)$ & $0.94(0.89 ; 0.96)$ \\
\hline
\end{tabular}

\subsection{Absolute intra-subject reliability}

Bland and Altman 95\% limits of agreement were measured and the scatter plots were analysed in all recording positions for all subjects. No systematic bias was seen (see Table 3).

The SRD measured did not change substantially over the different locations for either variable (see Table 4) indicating the stability of the measure. 
Table 3. Absolute reliability of the crackles' IDW and $2 \mathrm{CD}(\mathrm{n}=54)$. Values are from $\bar{d}$, mean; $\mathrm{SD}_{\text {diff, }}$ standard deviation of the differences; $S E \bar{d}$, standard error of the mean difference; $95 \%$

CI $\bar{d}, 95 \%$ confidence intervals of the mean difference; $95 \%$ LA, $95 \%$ limits of agreement. T: trachea; AR: anterior right; AL: anterior left; LR: lateral right; LL: lateral left; PR: posterior right; PL: posterior left.

\begin{tabular}{ccccccccccl}
\hline & $\begin{array}{c}\bar{d} \\
\mathrm{IDW} \\
(\mathrm{ms})\end{array}$ & $\begin{array}{c}\mathrm{SD}_{\mathrm{diff}} \\
\mathrm{IDW} \\
(\mathrm{ms})\end{array}$ & $\begin{array}{c}\bar{d} \\
2 \mathrm{CD} \\
(\mathrm{ms})\end{array}$ & $\begin{array}{c}\mathrm{SD}_{\mathrm{di}} \\
\text { 2CD } \\
(\mathrm{ms})\end{array}$ & $\begin{array}{c}S E \bar{d} \\
\mathrm{ID} \\
(\mathrm{ms})\end{array}$ & $\begin{array}{c}S E \bar{d} \\
2 \mathrm{CD}\end{array}$ & $\begin{array}{c}95 \% \mathrm{CI} \text { for } \\
\bar{d} \text { IDW } \\
(\mathrm{ms})\end{array}$ & $\begin{array}{c}95 \% \mathrm{CI} \text { for } \\
\bar{d} 2 \mathrm{CD} \\
(\mathrm{ms})\end{array}$ & $\begin{array}{c}95 \% \mathrm{LA} \\
\mathrm{IDW}(\mathrm{ms})\end{array}$ & $\begin{array}{c}95 \% \mathrm{LA} \\
2 \mathrm{CD}(\mathrm{ms})\end{array}$ \\
\hline $\mathrm{T}$ & -0.04 & 0.15 & -0.16 & 0.79 & 0.02 & 0.11 & $(-0.09 ; 0.01)$ & $(-0.40 ; 0.08)$ & $(-0.34 ; 0.26)$ & $(-1.74 ; 1.42)$ \\
$\mathrm{AR}$ & 0.04 & 0.24 & -0.17 & 1.12 & 0.03 & 0.15 & $(-0.03 ; 0.11)$ & $(-0.51 ; 0.17)$ & $(-0.44 ; 0.52)$ & $(-2.41 ; 2.07)$ \\
$\mathrm{AL}$ & -0.01 & 0.24 & -0.16 & 1.24 & 0.03 & 0.17 & $(-0.08 ; 0.07)$ & $(-0.54 ; 0.22)$ & $(-0.49 ; 0.48)$ & $(-2.64 ; 2.32)$ \\
$\mathrm{LR}$ & -0.01 & 0.22 & -0.14 & 0.97 & 0.03 & 0.13 & $(-0.08 ; 0.06)$ & $(-0.44 ; 0.16)$ & $(-0.45 ; 0.43)$ & $(-2.08 ; 1.8)$ \\
$\mathrm{LL}$ & -0.03 & 0.25 & -0.15 & 1.04 & 0.03 & 0.14 & $(-0.11 ; 0.05)$ & $(-0.47 ; 0.17)$ & $(-0.53 ; 0.47)$ & $(-2.23 ; 1.93)$ \\
$\mathrm{PR}$ & -0.04 & 0.28 & -0.11 & 0.94 & 0.04 & 0.13 & $(-0.13 ; 0.05)$ & $(-0.40 ; 0.18)$ & $(-0.6 ; 0.52)$ & $(-1.99 ; 1.77)$ \\
$\mathrm{PL}$ & -0.02 & 0.34 & 0.02 & 0.89 & 0.05 & 0.12 & $(-0.12 ; 0.08)$ & $(-0.25 ; 0.29)$ & $(-0.7 ; 0.66)$ & $(-1.76 ; 1.8)$ \\
\hline
\end{tabular}

Table 4. Smallest Real Difference of crackle IDW and 2CD $(n=54)$. Values are in milliseconds (ms). T: trachea; AR: anterior right; AL: anterior left; LR: lateral right; LL: lateral left; PR: posterior right; PL: posterior left).

\begin{tabular}{ccc}
\hline & SRD IDW $(\mathrm{ms})$ & SRD 2 CD $(\mathrm{ms})$ \\
\hline $\mathrm{T}$ & 0.30 & 1.57 \\
$\mathrm{AR}$ & 0.47 & 2.20 \\
$\mathrm{AL}$ & 0.46 & 2.42 \\
$\mathrm{LR}$ & 0.43 & 1.89 \\
$\mathrm{LL}$ & 0.49 & 2.05 \\
$\mathrm{PR}$ & 0.55 & 1.84 \\
$\mathrm{PL}$ & 0.66 & 1.74 \\
\hline
\end{tabular}

\section{Discussion}

This research has demonstrated that crackle IDW and 2CD show high intra-subject reliability in a clinical population over short time periods. The inter- and intra-subject reliability of crackle IDW and 2CD measured using CALSA has never been previously investigated. There is, however, a consensus that the inter and intra-observer reliability of the detection of added lung sounds among health professionals using tape-recorders, audio-files or standard auscultation is generally poor (Elphick et al., 2004, Kiyokawa et al., 2001).

The inter-subject reliability for both crackle variables studied (IDW and 2CD) in this research was found to be low, as shown by the uniformly significant ANOVA $(\mathrm{p}<0.001)$, indicating variability of mean crackles parameters across subjects. This is likely to be due to differences in demographic and anthropometric characteristics (Ploysongsang et al., 1991, Sanchez and Vizcaya, 2003), differences in pathology and varying acuity. Low inter-subject reliability of lung sounds has been previously reported (Ploysongsang et al., 1991, Sanchez and Vizcaya, 2003). Comparisons are difficult, however, because the samples studied by previous researchers involved only healthy subjects, and the lung sounds data were studied in the frequency domain where the spectral characteristics and patterns were analysed. In this research, lung sound data were collected from subjects with pathology and the reliability analysis was performed in the time domain to assess crackle parameters.

The intra-subject reliability was found to be 'excellent' with no systematic bias between repeated measures. Previous authors have reported high intra-subject reliability of lung sounds 
in healthy subjects alone (Mahagnah and Gavriely, 1994, Ploysongsang et al., 1991, Sanchez and Vizcaya, 2003) and in a joint study of healthy subjects and patients with fibrosing alveolitis (Mahagnah and Gavriely, 1994). However, these studies have analysed lung sounds in small samples of mainly healthy subjects, making comparisons difficult. The ICC for crackle parameters suggested 'excellent' reliability in all anatomical locations, in all subjects. However, lower values were found for the crackle IDW duration, possibly implying lower reliability. This could be because crackle IDW duration is a more difficult measure to obtain accurately, since the duration is very short and the precise point at which a crackle starts is difficult to determine (Hoevers and Loudon, 1990), increasing the measurement error. However, ICC in isolation should be interpreted with caution. Bland and Altman 95\% limits of agreement are independent of the true variability in the observations and therefore, complement the ICC analysis and provide detail regarding the nature of the observed intra-subject variability (Rankin and Stokes, 1998). The reliability assessed from Bland and Altman techniques was found to be acceptable, and no consistent bias was detected in any anatomical location for the two crackle parameters studied. Therefore, crackle characterisation using CALSA was deemed to be reproducible over short time periods.

In other studies regarding the assessment of the repeatability of lung sounds, the reliability of the spectral characteristics have been explored with analysis of variance (Sanchez and Vizcaya, 2003) or with analysis of variance and the coefficient of variation (Mahagnah and Gavriely, 1994, Ploysongsang et al., 1991, Sovijarvi et al., 1996). The use of the coefficient of variation to calculate reliability is not considered appropriate since it assumes that the largest test-retest differences will occur in individuals scoring the highest values on the test (Rankin and Stokes, 1998). The ICC and Bland and Altman 95\% limits of agreement have been recommended as more appropriate methods to assess reliability (Rankin and Stokes, 1998). In lung sound research, the ICC has been used by Schreur et al. (Schreur et al., 1992, Schreur et al., 1994) in studies involving patients with emphysema and normal subjects (Schreur et al., 1992) and in patients with asthma and normal lung function (Schreur et al., 1994). In both studies reliability was found to be 'satisfactory'. This analysis explored the reliability of lung sounds intensity (Schreur et al., 1992) and lung sounds intensity and wheezes characteristics (Schreur et al., 1994) and the recordings were performed in a sound proofed room. It has not been possible to compare the results obtained in our research directly with other investigations due to methodological differences. The SRD provides evidence of a real change that is not attributable to 'error' or 'noise'(Beckerman et al., 2001). In this study the SRD values, over short time periods, presented a similar range of values in the different anatomical locations, indicating the stability of the measure. The SRD values were much smaller (proportionally) for the 2CD than for the IDW. The lower SRD values imply higher reliability (Beckerman et al., 2001) which again suggests that the $2 \mathrm{CD}$ is a more stable and reliable measure than IDW.

Previous research involving CALSA has primarily been conducted in laboratory settings, with the use of a pneumotachograph to measure and control airflow. However, one of the disadvantages of the pneumotachograph is that patients have to breathe using a mouthpiece or a face mask. This causes patients discomfort and changes the breathing pattern, and so is rarely used clinically (Akre et al., 2000). Furthermore, patients with respiratory problems frequently also have neurological impairment or may have swallowing disorders, behaviour problems (unable to co-operate), physical deformities, or poor postural control which makes the use of these devices impractical. These aspects have also been acknowledged by other authors (Yadollahi and Moussavi, 2007). It is also known that crackle frequency depends on volume of inhaled air and not on rate of inhalation. The primary purpose of this study was to test the reliability of the methodology in a clinical setting, where control of airflow is often not practical and therefore we did not measure or control the volume of inhaled air.

\section{Conclusions}

These results indicate that crackle IDW and 2CD measures using CALSA are reproducible over short time periods in stable cystic fibrosis and bronchiectasis patients. Crackle 2CD is a more reliable and stable measure than crackle IDW. The findings suggest that any observed changes in CALSA are due to real changes and not to statistical variability. We have also demonstrated 
that CALSA can be used in a clinical setting, recording data via a standard digital stethoscope. It would be useful to evaluate this technique in other clinical environments, as it is a physiological measure that has several potential clinical applications in respiratory care.

\section{Acknowledgements}

The authors would like to thank all subjects for their involvement. We are also very grateful to the members of staff of the Respiratory Centre at Queen Alexandra Hospital, Portsmouth, UK. Finally, we would like to thank Escola Superior de Saúde da Universidade de Aveiro, Portugal, for supporting the first author (Alda Marques) during her PhD studies in the UK.

\section{Funding}

This study was funded by Fundação para a Ciência e Tecnologia (FCT), Portugal.

\section{References}

Akre, H., Borgersen, A. K., Mair, I. W. S. \& Skatvedt, O. (2000) Tracing air flow and diagnosing hypopnoeas in normal subjects. Physiological Measurement, 21, 221-227.

Ats, A. T. S. (1977) Update nomenclature for membership relation. ATS News, 3, 5-6.

Beckerman, H., Roebroeck, M. E., Lankhorst, G. J., Becher, J. G., Bezemer, P. D. \& Verbeek, A. L. M. (2001) Smallest real difference, a link between reproducibility and responsiveness. Quality of Life Research, 10, 571-578.

Bland, J. M. \& Altman, D. G. (1986) Statistical methods for assessing agreement between two methods of clinical measurement. Lancet, 1, 307-310.

Elphick, H. E., Lancaster, G. A., Solis, A., Majumdar, A., Gupta, R. \& Smyth, R. L. (2004) Validity and reliability of acoustic analysis of respiratory sounds in infants. Archives Disease Childhood, 89, 1059-1063.

Finch, E., Brooks, D., Stratford, O. W. \& Mayo, N. E. (2002) Physical Rehabilitation Outcome Measures, Lippincott Williams \& Wilkins.

Fleiss, J. (1986) Reliability of measurements, New York, John Wiley \& Sons.

Forgacs, P. (1978) Lung sounds, London, Bailliere Tindall.

Fredberg, J. \& Holford, S. K. (1983) Discrete lung sounds: crackles (rales) as stressrelaxation quadrupoles. Journal of Acoustic Society of America, 73, 1036-1046.

Hoevers, J. \& Loudon, R. G. (1990) Measuring crackles. Chest, 98, 1240-1243.

Hopkins, W. (2000) Measures of reliability in sports medice and science. Sports Medicine, 1, 1-15.

Kaisla, T., Sovijarvi, A., Piirila, P., Rajala, H.-M., Haltsonen, S. \& Rosqvist, R. (1991) Validated method for automatic detection of lung sound crackles. Medical \& Biological Engineering \& Computing, 29, 517-521.

Kiyokawa, H., Geenberg, M., Shirota, K. \& Pasterkamp, H. (2001) Auditory detection of simulated crackles in breath sounds. Chest, 119, 1886-1892.

Mahagnah, M. \& Gavriely, N. (1994) Repeatability of measurements of normal lung sounds. American Journal Respiratory Critical Care Medicine, 149, 477-481.

Marques, A., Bruton, A. \& Barney, A. (2006) Clinically useful outcome measures for physiotherapy airway clearance techniques: a review. Physical Therapy Reviews, 11, 299-307.

Miller, M. R., Hankinson, J., Brusasco, V., Burgos, F., Casaburi, R., Coates, A., Crapo, R., Enright, P., Grinten, C. P. M. V. D., Gustafsson, P., Jensen, R., Johnson, D. C., Macintyre, N., Mckay, R., Navajas, D., Pederson, O. F., Pellegrino, R., Viegi, G. \& Wanger, J. (2005) Standardisation of spirometry. European Respiratory Journal, 26, 319-338. 
Murphy, R. L. H., Bono, E. A. D. \& Davidson, F. (1989) Validation of an automatic crackle (rale) counter. American Review Respiratory Disease, 140, 1017-1020.

Murphy, R. L. H., Vyshedskiy, A., Power-Charnitsky, V.-A., Bana, D. S., Marinelli, P. M., Wong-Tse, A. \& Paciej, R. (2004) Automated Lung Sound Analysis in Patients with Pneumonia. Respiratory Care, 49, 1490-1497.

Nath, A. R. \& Capel, L. H. (1974) Inspiratory crackles and mechanical events of breathing. Thorax, 29, 695-698.

Piirila, P., Sovijarvi, A. R. A., Kaisla, T., Rajala, H.-M. \& Katila, T. (1991) Crackles in patients with fibrosing alveolitis, bronchiectasis, COPD, and heart failure. Chest, 99, 1076-1083.

Ploysongsang, Y., Iyer, V. K. \& Ramamoorthy, P. A. (1991) Reproducibility of the vesicular breath sounds in normal subjects. Respiration, 58, 158-162.

Rankin, G. \& Stokes, M. (1998) Reliability of assessment tools in rehabilitation: an illustration of appropriate statistical analyses. Clinical Rehabilitation, 12, 187199.

Sanchez, I. \& Vizcaya, C. (2003) Tracheal and lung sounds repeatability in normal adults. Respiratory Medicine, 97, 1257-1260.

Schreur, H. J. W., Sterk, P. J., Vanderschoot, J., Klink, H. C. J. V., Vollenhoven, E. V. \& Dijkman, J. H. (1992) Lung sound intensity in patients with emphysema and in normal subjects at standardised airflows. Thorax, 47, 674-679.

Schreur, H. J. W., Vanderschoot, J., Zwinderman, A. H., Dijkman, J. H. \& Sterk, P. J. (1994) Abnormal lung sounds in patients with asthma during episodes with normal lung function. Chest, 106, 91-99.

Sovijarvi, A. R. A., Malmberg, L. P., Charbonneau, G., Vanderschoot, J., Dalmasso, F., Sacco, C., Rossi, M. \& Earis, J. E. (2000a) Characteristics of breath sounds and adventitious respiratory sounds. European Respiratory Review, 10, 591-596.

Sovijarvi, A. R. A., Malmberg, P., Paajanen, E., Piirila, P., Kallio, K. \& Katila, T. (1996) Averaged and timed-gated spectral analysis of respiratory sounds Repeatability of spectral parameters in healthy men and in patients with fibrosing alveolitis. Chest, 109, 1283-1290.

Sovijarvi, A. R. A., Vanderschoot, J. \& Earis, J. E. (2000b) Computerized respiratory sound analysis (CORSA): recommended standards for terms and techniques. ERS Task force report. European Respiratory Review, 10, 595-649.

Vannuccini, L., Earis, J. E., Helisto, P., Cheetham, B. M. G., Rossi, M., Sovijarvi, A. R. A. \& Vandershoot, J. (2000) Capturing and preprocessing of respiratory sounds. European Respiratory Review, 10, 616-620.

Vannuccini, L., Rossi, M. \& Pasquali, G. (1998) A new method to detect crackles in respiratory sounds. Technology and Health Care, 6, 75-79.

Vyshedskiy, A., Alhashem, R. M., Paciej, R., Ebril, M., Rudman, I., Fredberg, J. J. \& Murphy, R. (2008) Mechanism of Inspiratory and Expiratory Crackles. Chest, 8.

Yadollahi, A. \& Moussavi, Z. M. K. (2007) Acoustical respiratory flow. IEEE Engineering in medicine and biology magazine. 